\title{
Synthesis of pole-zero assignment control law with minimum control input
}

M.-H. Tu

C.-M. Lin

Indexing terms: Control systems, Poles and zeros, Internal stability

\begin{abstract}
A new method of control system design is developed to simultaneously consider the internal stability, pole assignment, reference signal tracking and control input minimisation. A parametrised design algorithm of the pole-zero assignment control law is derived to achieve the desired goals. The free parameters are used to minimise the $L_{2}$-norm of the control input signal. Since the requirements of internal stability are satisfied, this design algorithm performs appropriately, even if the plant is unstable and/or is in a nonminimum phase. Examples are given to illustrate the validity of the design algorithm.
\end{abstract}

\section{Introduction}

In control systems, the pole dominates the transient response and the system stability, so there have been many studies [1-4] on the pole assignment design method. In addition, since the zero of a system plays an important role in the interaction between the system and its external environment, much research [5-8] has been carried out to achieve the reference signal tracking by assigning the appropriate zeros to a system. In addition to pole-zero assignment, another important control strategy is to minimise the control input signal of the control system. Optimal control theory [9] (more specifically, the linear quadratic regulator and linear quadratic Gaussian control theories) has considered the control input minimisation problem in the performance index. In Reference 10 , the minimum fuel control problem is investigated for a class of systems whose associated Lie algebra [11] is nilpotent. For plants with not 'too' large a parameter uncertainty, the result given in Reference 12 guarantees the minimisation of the control signal 'power' at the plant's input. For a minimum variance controller algorithm proposed by Mendes et al. [13], the weighting polynomials are calculated so as to assign the closed-loop poles of the system and to reduce the control signal's variances. A model reference control law has been proposed [14] to minimise a cost function including output error and weighting control input. The problems of pole assignment, signal tracking and input quadratic norm minimisation have already been investigated individually, however, a complete analysis of the internal stability,

Paper $8548 \mathrm{D}(\mathrm{C} 8)$, first received 14 th November 1990 and in revised form 18th October 1991

M.-H. Tu is with the Institute of Electronics, National Chiao-Tung University, 1001 Ta-Hsueh Road, Hsin-Chu 30050 Taiwan, Republic of China

C.-M. Lin is with the Chung-Shan Institute of Sciences and Technology, PO Box 90008-6-5, Lung-Tan, Tao-Yuan 32500, Taiwan, Republic of China pole assignment, reference signal tracking and control input minimisation of a system was not pursued in the above design methods. In this paper, some previously published ideas are collected to present a new method of control system design. The present contribution is the application of the conditions of previous results to construct a parametrised compensator assuring internal stability, pole assignment and reference signal tracking. The free parameters are then used to minimise the $L_{2}$-norm of the control input signal. Since internal stability [15] is ensured to avoid the unstable hidden mode of the control system, this design algorithm will perform appropriately even if the plant is unstable and/or is in a nonminimum phase.

\section{Problem formulation}

Consider the unity-feedback system shown in Fig. 1, where $P(s)$ and $C(s)$ denote the plant and controller,

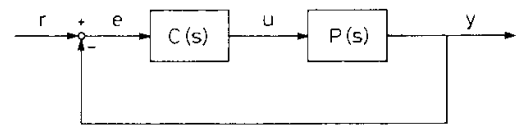

Fig. 1 Unity-feedback system

respectively. Let the plant be:

$$
P(s)=\frac{B(s)}{A(s)}
$$

where $A(s)$ and $B(s)$ are coprime polynomials.

The reference input is

$$
R(s)=\frac{N(s)}{M(s)}
$$

where $M(s)$ and $N(s)$ are coprime polynomials.

Define the sensitivity function $S(s)$ as

$$
S(s)=\frac{1}{1+P(s) C(s)}
$$

then the tracking error signal $E(s)$ can be obtained as

$$
E(s)=S(s) R(s)
$$

For a high-order reference signal (step, ramp, ..., etc.) tracking, the sensitivity function must have sufficient zeros to cancel the poles of $R(s)$ in the closed right-half plane (i.e. in $\operatorname{Re}[s] \geqslant 0$ ). Thus the zero assignment of $S(s)$ is very important in the reference signal tracking problem. The desired pole assignment of $S(s)$ also enables the system to achieve prespecified performance.

\section{Pole-zero assignment control law design}

To apply the design to any stable or unstable, minimum or nonminimum phase system, we first derive a pole-zero 
assignment control law which satisfies the internal stability. Let us factorise $A(s), B(s)$ and $M(s)$ as follows:

$$
\begin{aligned}
& A(s)=A_{+}(s) A_{-}(s) \\
& B(s)=B_{+}(s) B_{-}(s) \\
& M(s)=M_{+}(s) M_{-}(s)
\end{aligned}
$$

where $A_{+}(s), B_{+}(s)$ and $M_{+}(s)$ have all their zeros in $\operatorname{Re}[s] \geqslant 0$ while $A_{-}(s), B_{-}(s)$ and $M_{-}(s)$ have all their zeros in $\operatorname{Re}[s]<0$.

For the reference signal tracking and desired pole assignment, the sensitivity function must be of the form

$$
S(s)=\frac{W(s) M_{+}(s)}{G(s)}
$$

where $G(s)$ is a Hurwitz polynomial with desired closedloop poles and $W(s)$ is an undetermined polynomial which should be determined to satisfy the internal stability constraints.

\subsection{Definition 1}

The sensitivity function $S(s)$ is said to be internally stable if the closed-loop system of Fig. 1 is asymptotically stable for some choices of $C(s)$, i.e. there is no pole-zero cancellation between $C(s)$ and $P(s)$ in $\operatorname{Re}[s] \geqslant 0$ [15].

3.2 Lemma 1

The sensitivity function $S(s)$ is said to be internally stable if and only if all the following conditions hold [15]:

(i) $S(s)$ is analytic in $\operatorname{Re}[s] \geqslant 0$.

(ii) The numerator polynomial of $S(s)$ is divisible by $A_{+}(s)$.

(iii) The numerator polynomial of $1-S(s)$ is divisible by $B_{+}(s)$.

Remark: If $S(s)$ is internally stable, then from eqn. 3 , the corresponding controller can be derived as

$$
C(s)=\frac{1-S(s)}{P(s) S(s)}
$$

without worrying about any unstable pole-zero cancellation.

From condition (ii) of Lemma 1 , the numerator of $S(s)$ must contain $A_{+}(s)$, and from eqn. 8 , the numerator of $S(s)$ must also contain $M_{+}(s)$. Thus the numerator of $S(s)$ must contain the least common multiplier of $A_{+}(s)$ and $M_{+}(s)$, i.e.,

$$
S(s)=\frac{W(s) M_{+}(s)}{G(s)}=\frac{L(s) Z(s)}{G(s)}
$$

where $Z(s)$ is the least common multiplier of $A_{+}(s)$ and $M_{+}(s)$ while $L(s)$ is an undetermined polynomial. To satisfy the requirement of causality, the sensitivity function must be proper, i.e.

$$
\operatorname{deg}(G(s)) \geqslant \operatorname{deg}(L(s))+\operatorname{deg}(Z(s))
$$

where $\operatorname{deg}(*)$ denotes the degree of the polynomial *

From eqn. 9 , we have

$$
1-S(s)=\frac{G(s)-L(s) Z(s)}{G(s)}
$$

and from condition (iii) of Lemma 1, the numerator of $1-S(s)$ must contain $B_{+}(s)$. Thus we have

$$
H(s) \equiv G(s)-L(s) \mathcal{Z}(s)=B_{+}(s) F(s)
$$

where the number of undetermined parameters of $L(s)$ is greater than or equal to $\operatorname{deg}\left(B_{+}(s)\right)$, and $F(s)$ is an undetermined polynomial.

\subsection{Theorem 1}

The solution of $L(s)$ in eqn. 12 exists if and only if $M_{+}(s)$ is coprime with $B_{+}(s)$.

Proof: (If): Since $M_{+}(s)$ is coprime with $B_{+}(s)$, and $A_{+}(s)$ is coprime with $B_{+}(s)$, so that $Z(s)$ is coprime with $B_{+}(s)$. And since $G(s)$ is a Hurwitz polynomial and is coprime with $B_{+}(s)$, so that the solution of $L(s)$ in eqn. 12 exists. exists.

(Only if): In contrast, suppose $M_{+}(s)$ is not coprime with $B_{+}(s)$, so that $Z(s)$ and $B_{+}(s)$ must have a common factor with zeros in $\operatorname{Re}[s] \geqslant 0$. Since $G(s)$ is a Hurwitz polynomial and is coprime with $B_{+}(s)$, thus the solution of $L(s)$ in eqn. 12 does not exist. This contradicts the existence of the solution of $L(s)$ in eqn. 12 , so $M_{+}(s)$ must be coprime with $B_{+}(s)$. This completes the proof.

Remark: If $L(s)$ exists and the number of undetermined parameters of $L(s)$ is equal to $\operatorname{deg}\left(B_{+}(s)\right)$, then the solution of $L(s)$ in eqn. 12 is unique. By solving eqn. 12, we obtain $L(s)$ and $F(s)$. And then the controller can be derived as

$$
\begin{aligned}
C(s) & =\frac{1-S(s)}{P(s) S(s)} \\
& =\frac{A(s) B_{+}(s) F(s)}{B(s) L(s) Z(s)} \\
& =\frac{A_{-}(s) F(s)}{B_{-}(s) L(s) Q(s)}
\end{aligned}
$$

where

$$
Q(s)=\frac{Z(s)}{A_{+}(s)}
$$

It can be seen that if $A_{+}(s)$ and $M_{+}(s)$ have no common factor then $Q(s)=M_{+}(s)$; but if $A_{+}(s)$ contains $M_{+}(s)$ then $Q(s)=1$

Remark: From eqn. 13, for $C(s)$ to be proper, it is required that

$$
\begin{aligned}
\operatorname{deg}\left(B_{-}(s)\right)+\operatorname{deg}(L(s)) & +\operatorname{deg}(Q(s)) \\
& \geqslant \operatorname{deg}\left(A_{-}(s)\right)+\operatorname{deg}(F(s))
\end{aligned}
$$

The control input can be obtained from eqns. 2,9 and 13

$$
\begin{aligned}
U(s) & =C(s) S(s) R(s) \\
& =\frac{A_{-}(s) F(s)}{B_{-}(s) L(s) Q(s)} \frac{L(s) Z(s)}{G(s)} \frac{N(s)}{M(s)} \\
& =\frac{A(s) F(s) N(s)}{B_{-}(s) G(s) M(s)}
\end{aligned}
$$

Finally, the output $Y(s)$ can be derived from eqns. 2, 11 and 12 , i.e.

$$
\begin{aligned}
Y(s) & =(1-S(s)) R(s) \\
& =\frac{B_{+}(s) F(s) N(s)}{G(s) M(s)}
\end{aligned}
$$

If $L(s)$ exists and the number of undetermined parameters of $L(s)$ is greater than $\operatorname{deg}\left(B_{+}(s)\right)$, then the solution of $L(s)$ is not unique. This leads to an over-parametrised solution and the free parameters of $L(s)$ can be determined according to some specific performance criteria. In the following section, $L(s)$ is determined by minimising the $L_{2}$-norm of the control input signal $u(t)$.

IEE PROCEEDINGS-D, Vol 139, No. 3, MAY 1992 
In most practical control systems, the control input signal $u(t)$ has to be minimised to save the control energy and to avoid nonlinear saturation problems.

\subsection{Definition 2}

The $L_{2}$-norm of $u(t)$ is defined as [16]

$$
\|u\|_{2}=\left(\int_{-\infty}^{\infty} u^{2}(t) d t\right)^{1 / 2}
$$

If $u(t)$ is bounded for all value of $t$, is zero for $t<0$, and approaches zero at least as fast as $e^{-e t}$ as $t$ approaches infinity, where $\varepsilon$ is a small positive constant, then applying the Parseval theorem [17], we obtain

$$
\begin{aligned}
\|\boldsymbol{u}\|_{2} & =\left(\int_{0}^{\infty} u^{2}(t) d t\right)^{1 / 2} \\
& =\left(\frac{1}{2 \pi j} \int_{-j \infty}^{j \infty} U(s) U(-s) d s\right)^{1 / 2} \\
& \equiv\|U\|_{2}
\end{aligned}
$$

where $U(s)$ is the Laplace transform of $u(t)$, i.e., $U(s)$ is stable with poles in $\operatorname{Re}[s]<0$.

\subsection{Theorem 2}

If $A_{+}(s)$ is divisible by $M_{+}(s)$ and $U(s)$ is a strictly proper rational function then $\|U\|_{2}$ in eqn. 19 is finite.

Proof: Since $A_{+}(s)$ is divisible by $M_{+}(s)$, and $B_{-}(s)$ and $G(s)$ are Hurwitz polynomials, from eqn. $16, U(s)$ is a stable function. Since $U(s)$ is a strictly proper rational function, $\|U\|_{2}$ is finite [18]. This completes the proof.

If $A_{+}(s)$ is divisible by $M_{+}(s)$, and $U(s)$ is a strictly proper rational function with free parameters, then we can use the algorithm given in Reference 18 to obtain $\|U\|_{2}$ in terms of free parameters. Furthermore, the minimum value of $\|U\|_{2}$ and the corresponding free parameters can be obtained by solving the simultaneous equations of partial derivatives of $\|U\|_{2}$ with respect to these free parameters.

Remarks:

(i) If the reference input $R(s)$ is stable, then $U(s)$ is stable, $\|U\|_{2}$ is finite as long as $U(s)$ is strictly proper. Since the closed-loop system is also stable, stable reference signal tracking can always be achieved. In this case $Z(s)$ is equal to $A_{+}(s)$, and the constraint in Theorem 2 , that $A_{+}(s)$ is divisible by $M_{+}(s)$, can be relaxed, thus the design goals are reduced to assuring the internal stability, pole assignment and control input minimisation. If necessary, the design goals can be extended, for example, to minimise a cost function including the tracking error and the control input $[9,14,17]$.

(ii) For high-order reference signal tracking, if $A_{+}(s)$ does not fully contain $M_{+}(s)$, one approach is to first augment the plant at its input or output with a transfer function $1 / Q(s)$ if this is realisable in the system [9, Section 4.3]. Otherwise, the denominator polynomial of $C(s)$ must contain $Q(s)$ as in eqns. 13 and 14 , so that the high-order reference signal tracking with stable closedloop system can be achieved despite the fact that $u(t)$ is a nondecaying function. In most practical systems, for high-order reference signal tracking, the considered time is finite, thus $\|u\|_{2}$ is still finite. Hence the control input minimisation is also significant in this case.

From the above analysis, we obtain the following design algorithm for pole-zero assignment control law with minimum control input.

IEE PROCEEDINGS-D, Vol. I39, No. 3, MAY 1992
Step 1: Perform the factorisation of $A(s), B(s)$ and $M(s)$ as in eqns. $5-7$, respectively, and determine $Z(s)$.

Step 2: From eqn. 12, solve $L(s)$ and $F(s)$ with free parameters, where $G(s)$ is determined by the desired closed-loop poles.

Step 3: Obtain $U(s)$ with free parameters as in eqn. 16, and solve the minimum of $\|U\|_{2}$ to obtain the values of free parameters (i.e., $L(s)$ and $F(s)$ are solved).

Step 4: Obtain the controller $C(s)$ from eqn. 13.

\section{Examples}

Two examples are given to illustrate the validity of the proposed algorithm.

5.1 Example 1

For a given unstable, nonminimum phase plant:

$$
P(s)=\frac{s-5}{s(s-1)}
$$

a pole-zero assignment control law and a minimum control input pole-zero assignment control law, respectively, are designed for the closed-loop system to track the unit step reference input signal, and to have the closed-loop poles assigned at $-1 \pm j 1,-2$ and -3 .

Solution: (a) Pole-zero assignment control law: In this system, $A_{+}(s)=s(s-1)$ contains $M_{+}(s)=s$, thus $U(s)$ is stable. The poles of $S(s)$ are assigned at $-1 \pm j 1,-2$ and -3 . To satisfy conditions (ii) and (iii) of Lemma 1 and the requirements of causality and a proper controller, since $B_{+}(s)=s-5$, we choose $L(s)=s^{2}+s+l_{1}$ with one undermined parameter. Then

$$
\begin{aligned}
& \qquad \begin{aligned}
H(s) & =\left(s^{2}+2 s+2\right)(s+2)(s+3)-\left(s^{2}+s+l_{1}\right) s(s-1) \\
& =(s-5) F(s) \\
H(5) & =0
\end{aligned} \\
& \text { then } \\
& l_{1}=73.6 \\
& F(s)=7 s^{2}-19.6 s-2.4
\end{aligned}
$$

thus

$$
\begin{aligned}
& C(s)=\frac{7 s^{2}-19.6 s-2.4}{s^{2}+s+73.6} \\
& U(s)=\frac{(s-1)\left(7 s^{2}-19.6 s-2.4\right)}{\left(s^{2}+2 s+2\right)(s+2)(s+3)} \\
& Y(s)=\frac{(s-5)\left(7 s^{2}-19.6 s-2.4\right)}{s\left(s^{2}+2 s+2\right)(s+2)(s+3)}
\end{aligned}
$$

and

$$
\|U\|_{2}=2.8988
$$

Solution: (b) Minimum control input pole-zero assignment control law: Since $\operatorname{deg}\left(B_{+}(s)\right)=1$, we choose $L(s)=s^{2}$ $+l_{1} s+l_{2}$ with two undetermined parameters. Then

$$
\begin{aligned}
H(s)= & \left(s^{2}+2 s+2\right)(s+2)(s+3) \\
& -\left(s^{2}+l_{1} s+l_{2}\right) s(s-1) \\
= & (s-5) F(s) \\
H(5)=0 & \\
l_{2}= & 78.6-5 l_{1} \\
F(s)= & \left(8-l_{1}\right) s^{2}+\left(l_{1}-20.6\right) s-2.4
\end{aligned}
$$


Thus

$$
U(s)=\frac{(s-1)\left(\left(8-l_{1}\right) s^{2}+\left(l_{1}-20.6\right) s-2.4\right)}{\left(s^{2}+2 s+2\right)(s+2)(s+3)}
$$

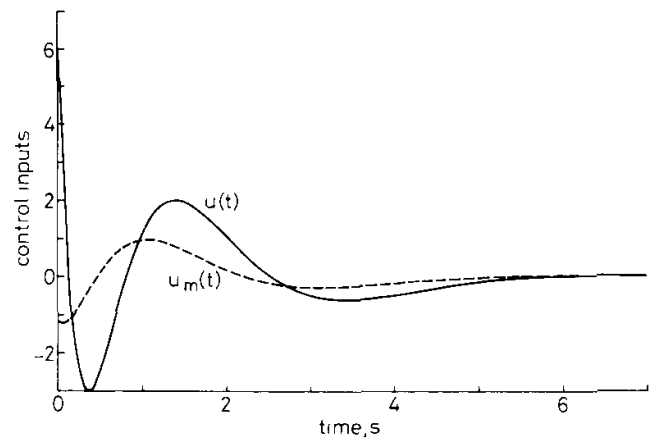

Fig. 2 Control inputs against time for example 1

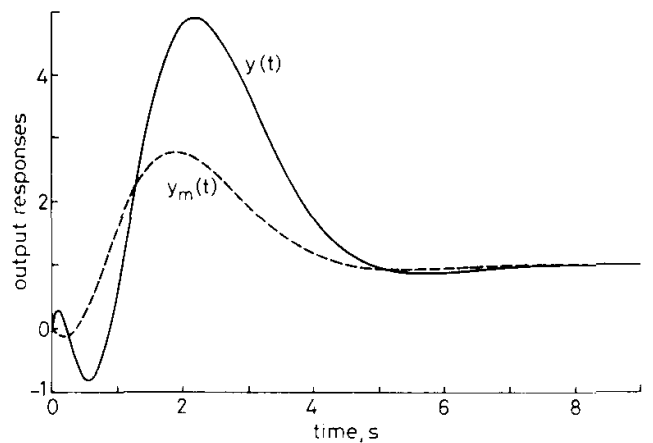

Fig. 3 Outputs against time for example I

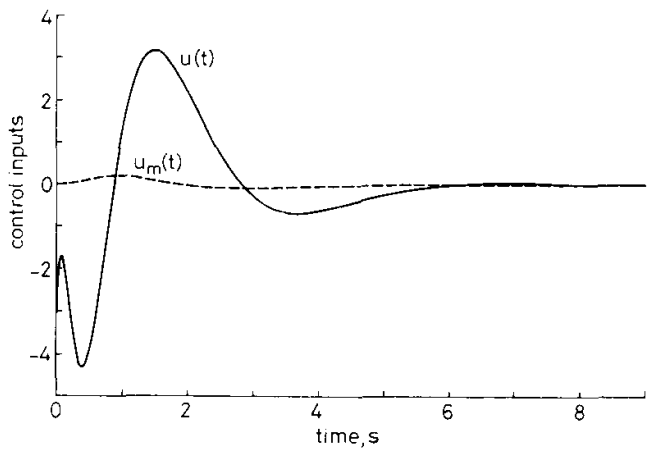

Fig. 4 Control inputs against time for example 2

$\|U\|_{2}$ is minimised at $l_{1}=9.1983$. Thus the minimum control input

$$
\begin{aligned}
& U_{m}(s)=\frac{(s-1)\left(-1.198 s^{2}-11.402 s-2.4\right)}{\left(s^{2}+2 s+2\right)(s+2)(s+3)} \\
& \left\|U_{m}\right\|=1.1079
\end{aligned}
$$

And the controller $C_{m}(s)$ and output $Y_{m}(s)$ corresponding to $U_{m}(s)$ can be obtain as

$$
\begin{aligned}
C_{m}(s) & =\frac{-1.198 s^{2}-11.402 s-2.4}{s^{2}+9.198 s+32.61} \\
Y_{m}(s) & =\frac{(s-5)\left(-1.198 s^{2}-11.402 s-2.4\right)}{s\left(s^{2}+2 s+2\right)(s+2)(s+3)}
\end{aligned}
$$

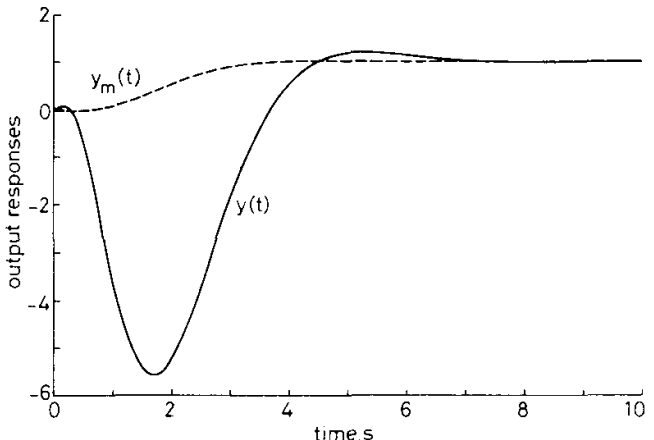

Fig. 5 Outputs against time for example 2

The control inputs $u(t)$ and $u_{m}(t)$ are shown in Fig. 2. The outputs $y(t)$ and $y_{m}(t)$ are shown in Fig. 3.

\subsection{Example 2}

A model of the flexible robot arm is represented as [19]

$$
P(s)=\frac{-4.9065(s-8.5568)(s+8.4294)}{s(s+0.2)\left((s+0.17)^{2}+(11.79)^{2}\right)}
$$

A pole-zero assignment control law and a minimum control input pole-zero assignment control law, respectively, are designed for the closed-loop system of the robot arm to track the unit step reference input signal, and to have the closed-loop poles assigned at $-1 \pm j 1$, -2 and -3 .

Solution (a) Pole-zero assignment control law: In this system, $A_{+}(s)=s$ contains $M_{+}(s)=s$, thus $U(s)$ is stable The poles of $S(s)$ are assigned at $-1 \pm j 1,-2$ and -3 , i.e., $G(s)=s^{4}+7 s^{3}+18 s^{2}+22 s+12$. To satisfy conditions (ii) and (iii) of Lemma 1 and the requirements of causality and a proper controller, since $B_{+}(s)=$ $s-8.5568$, we choose $L(s)=s^{3}+7 s^{2}+s+l_{1}$ with one undetermined parameter. Then

$$
\begin{aligned}
& \qquad \begin{aligned}
H(s)= & \left(s^{2}+2 s+2\right)(s+2)(s+3) \\
& -\left(s^{3}+7 s^{2}+s+l_{1}\right) s \\
= & (s-8.5568) F(s)
\end{aligned} \\
& H(8.5568)=0 \\
& \text { then } \quad \\
& l_{1}=168.86 \\
& F(s)=17 s-1.4024
\end{aligned}
$$

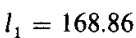

thus

$$
\begin{aligned}
& C(s)=\frac{(s+0.2)\left((s+0.17)^{2}+(11.79)^{2}\right)(17 s-1.4024)}{-4.9065(s+8.4294)\left(s^{3}+7 s^{2}+s+168.86\right)} \\
& U(s)=\frac{(s+0.2)\left((s+0.17)^{2}+(11.79)^{2}\right)(17 s-1.4024)}{-4.49065(s+8.4294)\left(s^{2}+2 s+2\right)(s+2)(s+3)} \\
& Y(s)=\frac{(s-8.5568)(17 s-1.4024)}{s\left(s^{2}+2 s+2\right)(s+2)(s+3)}
\end{aligned}
$$

IEE PROCEEDINGS-D, Vol. I39, No. 3, MAY 1992 
and

$$
\|U\|_{2}=4.1354
$$

Solution (b) Minimum control input pole-zero assignment control law: Since $\operatorname{deg}\left(B_{+}(s)\right)=1$, we choose $L(s)=s^{3}$ $+7 s^{2}+l_{1} s+l_{2}$ with two undetermined parameters. Then

$$
\begin{aligned}
H(s)= & \left(s^{2}+2 s-2\right)(s+2)(s+3) \\
& -\left(s^{3}+7 s^{2}+l_{1} s+l_{2}\right) s \\
= & (s-8.5568) F(s) \\
& 0 \\
l_{2}= & 177.42-8.5568 l_{1} \\
F(s)= & \left(18-l_{1}\right) s-1.4024
\end{aligned}
$$$$
H(8.5568)=0
$$

thus

$$
U(s)=\frac{(s+0.2)\left((s+0.17)^{2}+(11.79)^{2}\right)\left(\left(18-l_{1}\right) s-1.4024\right)}{-4.9065(s+8.4294)\left(s^{2}+2 s+2\right)(s+2)(s+3)}
$$

$\|U\|_{2}$ is minimised at $l_{1}=18$. Thus the minimum control input

$$
\begin{aligned}
U_{m}(s) & =\frac{0.2858(s+0.2)\left((s+0.17)^{2}+(11.79)^{2}\right)}{(s+8.4294)\left(s^{2}+2 s+2\right)(s+2)(s+3)} \\
\left\|U_{m}\right\|_{2} & =0.2107
\end{aligned}
$$

And the controller $C_{m}(s)$ and output $Y_{m}(s)$ corresponding to $U_{m}(s)$ can be obtained as

$$
\begin{aligned}
& C_{m}(s)=\frac{0.2858(s+0.2)\left((s+0.17)^{2}+(11.79)^{2}\right)}{(s+8.4294)\left(s^{3}+7 s^{2}+18 s+23.3976\right)} \\
& Y_{m}(s)=\frac{-1.4024(s-8.5568)}{s\left(s^{2}+2 s+2\right)(s+2)(s+3)}
\end{aligned}
$$

The control inputs $u(t)$ and $u_{m}(t)$ are shown in Fig. 4. The outputs $y(t)$ and $y_{m}(t)$ are shown in Fig. 5.

\section{Conclusions}

We have proposed a pole-zero assignment control law with minimum control input to simultaneously achieve internal stability, pole assignment, reference signal tracking and control input minimisation. Since the requirements of internal stability are satisfied, this design algorithm performs appropriately, even if the plant is unstable and/or is in a nonminimum phase. From the examples, we can see that when the control inputs are minimised, the outputs are more satisfactory (with less overshoot and undershoot).

\section{Acknowledgment}

The authors wish to thank the reviewers for their comments and suggestions.

\section{References}

1 WOLOVICH, W.A.: 'Linear multivariable systems' (SpringerVerlag, New York, 1974)

2 KAILATH, T.: 'Linear systems' (Prentice-Hall, Englewood Cliffs, NJ, 1980)

3 CHEN, C.-T.: 'Linear system theory and design' (Holt, Rinehart and Winston, New York, 1984)

4 ASTROM, K.J., and WITTENMARK, B.: 'Computer controlled systems' (Prentice-Hall, Englewood Cliffs, NJ, 1990)

5 FRANCIS, B.A.: 'The multivariable servomechanism problem from the input-output viewpoint', IEEE Trans., 1977, AC-22, pp. 322-328 BENGTSSON, B.: 'Output regulation and internal models: a frequency domain approach', Automatica, 1977, 13, pp. 333-345

7 WOLOVICH, W.A.: 'Multipurpose controllers for multivariable systems', IEEE Trans., 1981, AC-26, pp. 162-170

8 SAEKS, R and MURRAY, J ' 'Feedback system design: the tracking and disturbance rejection problem', IEEE Trans., 1981, AC-26, ing and disturb

9 ANDERSON, B.D.O., and MOORE, J.B.: 'Linear optimal control' (Prentice-Hall, Englewood Cliffs, NJ, 1989)

10 LIU, J.S., YUAN, K., and LIN, W.S.: 'On minimum-fuel control of affine nonlinear systems', IEEE Trans., 1989, AC-34, pp. 767--770

11 SAGLE, A.A., and WALDE, R.E.: 'Introduction to Lie groups and Lie algebras' (Academic, New York, 1973)

12 DICKMAN, A., and SIVAN, R.: 'On the robustness of multivariable linear feedback systems', IEEE Trans., 1985, AC-30, pp. 401-404

13 MENDES, R.S., AMARAL, W.C., and LATRE, L.G.: 'Determination of weighting polynomials in generalised minimum variance controllers', IEE Proc. D., Control Theory \& Appl., 1988, 135, (1), pp. 21-27

14 HWANG, C.L., and CHEN, B.S.: 'Model reference adaptive control via the minimisation of output error and weighting control input', IEE Proc. D. Control Theory \& Appl., 1989, 136, (5), pp. 231-237

15 YOULA, D.C, BONGIORNO, J.J., and JABR, H.A.: 'Mordern Wiener-Hopf design of optimal controllers, part $I$ : the single-inputoutput case', IEEE Trans., 1976, AC-21, pp. 3-13

16 MACIEJOWSKI, J.M.: 'Multivariable feedback design' (AddisonWesley, New York, 1990)

17 CHANG, S.S.L.: 'Synthesis of optimum control systems' (Maple Press, York, PA, 1961)

18 ÅSTRØ̊M, K.J.: 'Introduction to stochastic control theory' (Academic Press, New York, 1970)

19 CANNON, R.H. Jr., and SCHMITZ, E.: 'Initial experiments on the end-point control of a flexible one-link robot', Int. J. Robot. Res., 1984,3 , pp. 62-75 